\title{
Phase field theory of interfaces and crystal nucleation in a eutectic system of fcc structure: II. Nucleation in the metastable liquid immiscibility region
}

\author{
Gyula I. Tóth \\ Research Institute for Solid State Physics and Optics, P.O. Box 49, H-1525 Budapest, Hungary \\ László Gránásy \\ Brunel Centre for Advanced Solidification Technology, Brunel University, Uxbridge, Middlesex UB8 3PH, \\ United Kingdom and Research Institute for Solid State Physics and Optics, P.O. Box 49, H-1525 \\ Budapest, Hungary
}

(Received 8 September 2006; accepted 1 June 2007; published online 21 August 2007)

\begin{abstract}
In the second part of our paper, we address crystal nucleation in the metastable liquid miscibility region of eutectic systems that is always present, though experimentally often inaccessible. While this situation resembles the one seen in single component crystal nucleation in the presence of a metastable vapor-liquid critical point addressed in previous works, it is more complex because of the fact that here two crystal phases of significantly different compositions may nucleate. Accordingly, at a fixed temperature below the critical point, six different types of nuclei may form: two liquid-liquid nuclei: two solid-liquid nuclei; and two types of composite nuclei, in which the crystalline core has a liquid "skirt," whose composition falls in between the compositions of the solid and the initial liquid phases, in addition to nuclei with concentric alternating composition shells of prohibitively high free energy. We discuss crystalline phase selection via exploring/ identifying the possible pathways for crystal nucleation. (C) 2007 American Institute of Physics.
\end{abstract}

[DOI: $10.1063 / 1.2752506]$

\section{INTRODUCTION}

In the previous part of our phase field study of crystal nucleation in eutectic systems, we have addressed regions, where the liquid phase is stable against liquid-liquid phase separation. However, eutectic systems contain a metastable (MS) liquid-liquid coexistence region usually well below the eutectic temperature [see, e.g., Fig. 1 of Part I (Ref. 1)]. This metastable liquid-liquid coexistence might be expected to interact with crystal nucleation in a way analogous to the effect of metastable fluid-fluid coexistence on crystallization in single component fluids, addressed in depth in several papers. ${ }^{2-5}$ Since recent advances have been reviewed by Sear in Ref. 6, we recall here only briefly the main findings. Previous work done on single component fluids with metastable liquid-vapor critical point revealed that crystal nucleation is enhanced significantly at the critical point or slightly above. $^{2-5}$ This enhancement of the nucleation rate near the MS critical point has been indicated by atomistic simulations $^{2}$ and density functional theory ${ }^{3}$ (thought the predictions were questioned in some papers ${ }^{4}$ ) and has been confirmed by experiment. ${ }^{5}$ Atomistic simulations indicate that the effect may depend on the distance between the critical and melting points. ${ }^{7}$ Numerous experiments show that crystallization takes place indeed in a narrow temperature range in systems with such a phase diagram. ${ }^{8}$ This behavior is common in a broad variety of systems of short range interaction, including proteins/colloids. ${ }^{8,9}$ (Analogous situation exists in binary alloys with a metastable liquid-liquid miscibility gap. ${ }^{10}$ )
Atomistic simulations and density functional studies have revealed two significantly different pathways for crystal nucleation under such conditions: "solidlike" and "liquidlike" clusters, where in the latter the crystal core is surrounded by a liquid "skirt" of a density, which falls between densities of the solid core and the initial liquid. ${ }^{2,3}$ The latter type resembles the composite nuclei observed in model systems of a free energy composed of three parabolic minima. ${ }^{11}$ Indeed, in the metastable coexistence region, the free energy surface of these system has three minima: two for the fluid phases and one for the crystal. It may also be expected that deep in the metastable coexistence region, the homogeneous liquid phase becomes unstable with respect to phase separation into two fluid phases of different densities. In mean field theories, including the density functional approach used in Ref. 3, under such conditions transition to the two fluid phases occurs via spinodal decomposition. ${ }^{12}$ Experiments on polymer crystallization provide evidence that the morphology of the polymer crystals is indeed dominated by the interplay of crystallization and liquid-liquid demixing. ${ }^{13}$ Interaction between phase separation and transient nucleation has been studied experimentally on binary oxide glasses, though far from the critical point. ${ }^{14}$ A strong interaction has been revealed at early stages of crystal nucleation: phase separation enhanced nucleation. ${ }^{14}$ Despite previous work, a complete mapping of the possible nucleation pathways as a function of temperature and density inside the metastable fluidfluid coexistence region is yet unavailable. In the case of globular proteins, comparison with density functional calculations show that classical nucleation theory is invalid not 
only in the vicinity of the metastable critical point but also close to the liquidus line. ${ }^{3(d)}$ Another class of systems that has some similarities is that of colloidal mixtures of short range interaction, where two solid phases of different densities coexist. ${ }^{15}$ Nucleation properties incompatible with the classical nucleation theory have been reported.

In spite of the apparent similarities between the systems mentioned above and the eutectic system, an obvious difference is that in the latter, there are two solid phases to which the system can crystallize. Accordingly, instead of three, the free energy surface now has four minima: two for the liquid phases and two for the solid solutions. Thus the results obtained for crystal nucleation in the presence of metastable fluid-fluid or solid-solid critical point would not immediately apply for crystal nucleation at the MS liquid-liquid critical point of eutectic systems. Besides its theoretical interest, investigation of crystal nucleation in the MS liquid-liquid region is further stressed by the practical importance of eutectic systems. Identification of possible nucleation pathways may help us to understand phase selection and factors that control the microstructure.

Herein, we apply the phase field approach described in Part I to investigate crystal nucleation pathways as functions of temperature and composition inside the metastable liquidliquid coexistence region and in the vicinity of the respective critical point of the $\mathrm{Ag}-\mathrm{Cu}$ system. While in this work we address homogeneous nucleation; our study can be extended for heterogeneous nucleation along the lines described in Ref. 16.

This second part of our study of crystal nucleation in eutectic systems (Part II, henceforth) is structured as follows. In Sec. II, we briefly review specific questions emerging when applying the phase field approach to addressing metastable liquid-liquid coexistence, composite nuclei, etc., and the numerical methods needed to solve the appropriate EulerLagrange equations. The materials properties are presented in Sec. III. Section IV reviews our results for the planar liquid-liquid interface, the liquid-liquid nuclei, and the identification of various pathways of crystal nucleation, including composite nuclei in which the crystal-like core is surrounded by a liquid layer of composition falling between the compositions of the solid core and of the initial liquid. Finally, our findings are summarized in Sec. V.

\section{APPLIED MODELS}

Since the models used here are the same or similar to those used in Part I, we present only those details that are needed for a clear understanding of what has been done.

\section{A. Phase field theory for liquid-liquid interfaces and liquid phase separation}

The formulation used here is analogous to the one applied for studying solid-solid coexistence and nucleation in Part I: Below the metastable critical point two liquid phases (L1, L2) coexist $(\phi=1)$. The free energy of the respective inhomogeneous liquid-liquid system reads as

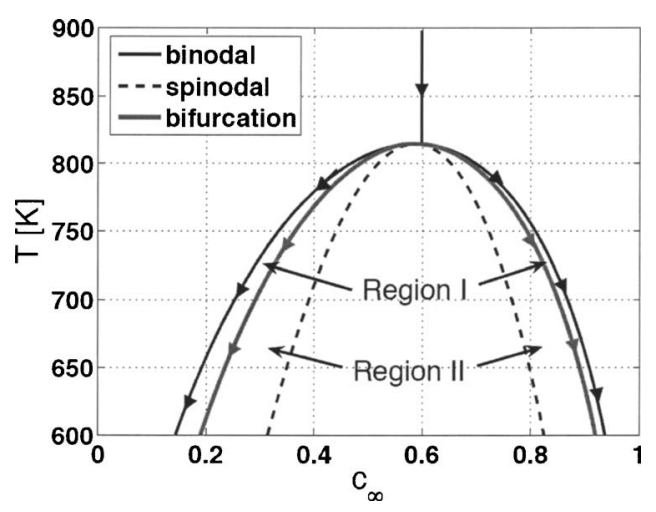

FIG. 1. Metastable liquid-liquid coexistence region in the phase diagram of the $\mathrm{Ag}-\mathrm{Cu}$ system, computed using the free energy functions from CALPHADtype calculations of Ref. 19. Note that coexistence line (thin solid), the spinodal line (dashed solid), and the bifurcation line (heavy gray line). Regions I and II may show different nucleation properties. For explanation, see the text.

$$
F_{L}=\int d^{3} r\left\{\frac{\varepsilon_{c, L}^{2}}{2}(\nabla c)^{2}+f_{L}(c)+\lambda_{L} c\right\},
$$

while the following Cahn-Hilliard-type Euler-Lagrange (EL) equation applies,

$$
\varepsilon_{c, L}^{2} \nabla^{2} c=-\frac{1}{2} \frac{\partial \varepsilon_{c, L}^{2}}{\partial c}(\nabla c)^{2}+\frac{\partial f_{L}}{\partial c}+\lambda_{L}
$$

For planar interfaces the latter needs to satisfy that L1 and L2 solutions of equilibrium composition exist in the far fields $(z \rightarrow \pm \infty)$. The Lagrange multiplier can be then identified as $\lambda_{L}=-\partial f_{L} / \partial c(z= \pm \infty)$. After trivial algebraic manipulations, the liquid-liquid interface thickness and the respective surface tension can be determined, as described in Ref. 17.

\section{B. Phase field theory for crystal nucleation and crystal-liquid interfaces}

The model used here for crystal nucleation is exactly the same as the phase field theory (PFT) with Ginzburg-Landau free energy presented in Part I. However, to be able to find the considerably more complex and multiple solutions of the Euler-Lagrange equations, we use here a different numerical scheme: A relaxation technique described in Ref. 18 has been applied. This led to a considerably faster finding of the solutions than the iteration scheme described in Part I.

\section{PHYSICAL PROPERTIES}

Here we used exactly the same physical data for the $\mathrm{Ag}-\mathrm{Cu}$ system as in Part 1 (see Table 1 of Ref. 1). Notably, the interaction parameter used in calculating the coefficient of the square-gradient term for liquid-liquid coexistence has been identified as $\Omega^{L}=A_{L}+A 1_{L}(1-2 c)$ using the notations of Table 1 of Ref. 19. The liquid-liquid immiscibility region of the phase diagram including the coexistence and spinodal lines calculated using these data is presented in Fig. 1. The respective liquid-liquid critical temperature is $T_{c}^{\mathrm{LL}}=814.5 \mathrm{~K}$. 


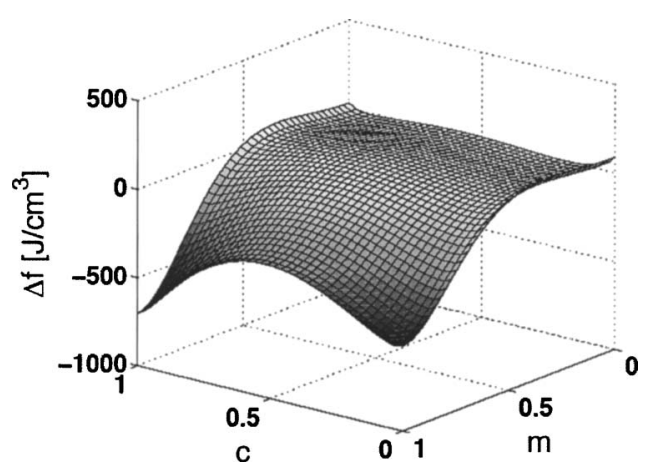

FIG. 2. Free energy density surface at $T=750 \mathrm{~K}$ in the phase field theory, counted relative to a homogeneous liquid of the critical composition. Note that at this temperature, besides having two stable solid compositions in equilibrium (at $\phi=0$ ), two metastable liquid compositions can also be in equilibrium with each other (at $\phi=1$ ).

The free energy surfaces typical to $T<T_{c}^{L L}$ yield stable solidsolid coexistence and metastable liquid-liquid coexistence (see Fig. 2).

\section{RESULTS AND DISCUSSION}

\section{A. Equilibrium liquid-liquid interfaces and nuclei}

Since these results are consistent with the Cahn-Hilliard theory, and are well known, here we review them briefly for the sake of completeness. The temperature dependencies of the surface tension and the interface thicknesses are presented in Figs. 3(a) and 3(b). The surface tension tends to zero, while the interface thickness diverges at the critical temperature with the appropriate mean field critical exponents. ${ }^{12,17}$ Between the coexistence and spinodal lines of the metastable liquid-liquid miscibility region, phase separation takes place via liquid-liquid nucleation. ${ }^{12,20}$ Examples of the respective radial concentration profiles at $T=750 \mathrm{~K}$ are shown in Fig. 4(a) together with the corresponding nucleation barrier heights [see Fig. 4(b)]. Inside the spinodal line phase separation takes place via spinodal decomposition. ${ }^{12,20}$ These nuclei are expected to interact with crystal nucleation. We treat the spinodal region and the region between the spin-
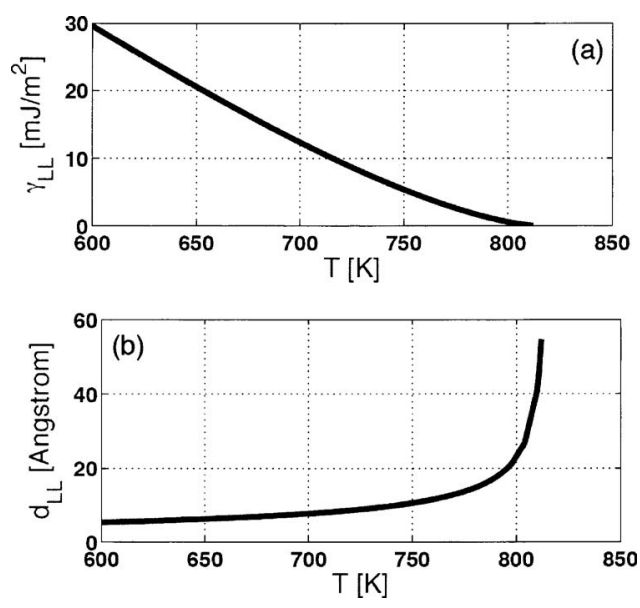

FIG. 3. Properties of the metastable planar liquid-liquid interface as a function of temperature: (a) surface tension; (b) 10\%-90\% interface thickness. Convergence of the former to zero and the divergence of the latter happen with the appropriate mean field critical exponents.
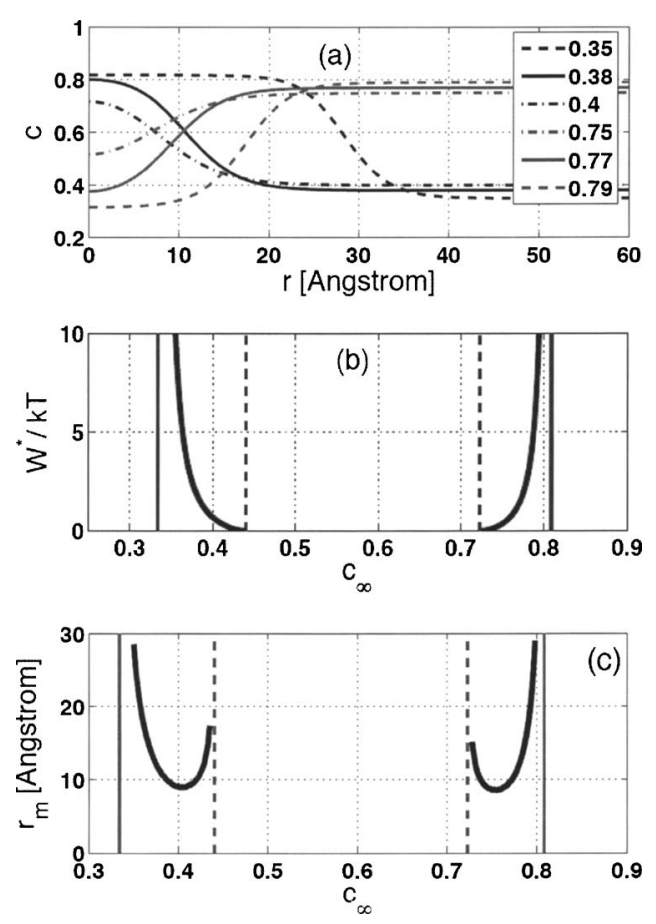

FIG. 4. Properties of liquid-liquid nuclei as a function of initial liquid composition at $T=750 \mathrm{~K}$ : (a) Concentration profiles (black lines: $\mathrm{Cu}$ rich liquid nuclei forming in initial liquid compositions falling between the coexistence and spinodal lines to the left of the critical composition; gray lines; Ag rich liquid nuclei forming on the opposite side of the phase diagram). (b) Nucleation barrier for liquid-liquid nuclei as a function of the initial liquid composition (the vertical solid and dashed lines indicate the coexistence and spinodal compositions, respectively). (c) Critical radii of liquid-liquid nuclei defined via the Gibbs surface for the concentration as a function of the initial liquid concentration.

odal and coexistence lines separately. It is also expected that in the close vicinity of the critical point, the metastable liquid phase separation slows down (critical slowing down).

\section{B. Nucleation pathways}

In this subsection, we investigate possible nucleation pathways available near the critical point, inside the spinodal regime, and between the spinodal and coexistence lines. While the behavior observed at the critical point closely follows previous results from other continuum models, we observe a fairly complex behavior inside the metastable liquidliquid coexistence region due to the larger number of nucleation pathways identified in this work.

\section{Crystal nucleation near the critical point}

First, we investigate the nucleation barrier in the vicinity of the MS liquid-liquid critical point. Previous work by Shiryayev and Gunton suggested ${ }^{3(\mathrm{~d})}$ that along the constant driving force line, one should observe a minimum in the nucleation barrier. Due to the two types of solid solutions appearing in the $\mathrm{Ag}-\mathrm{Cu}$ phase diagram, in our case, there are two such lines that intersect each other at the critical point: one for $\mathrm{Cu}$ rich and another for $\mathrm{Ag}$ rich nuclei [see Fig. 5(a)]. Indeed, we observe a shallow minimum in $W / k T$ slightly above $T_{c}$ in both cases [see Figs. 5(b) and 5(c)]. These results indicate that similar to other continuum theories, such as the density functional theory, ${ }^{3}$ the PFT predicts an enhanced 

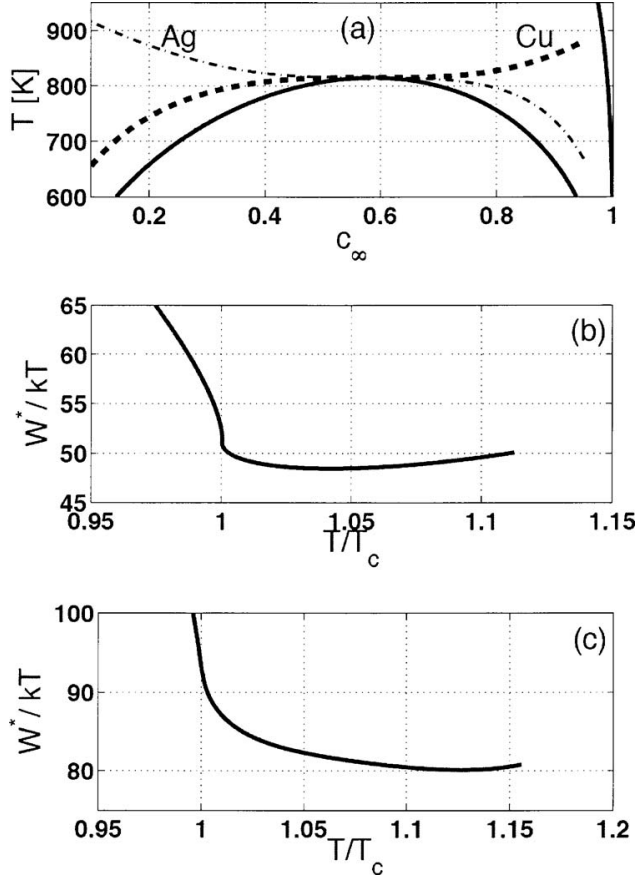

FIG. 5. Variation of the nucleation barrier for the $\mathrm{Cu}$ and $\mathrm{Ag}$ rich crystal nuclei along the constant driving force lines that cross the critical point. (a) The corresponding $c(T)$ trajectories (dashed and dash-dot lines, respectively). For comparison the metastable liquid-liquid coexistence line is also shown (solid line). (b) Barrier height vs reduced temperature for $\mathrm{Cu}$ rich nuclei. (c) Barrier height vs reduced temperature for the Ag rich nuclei.

nucleation rate near to the critical temperature along such lines. It is interesting to compare the $\phi$ vs $c$ trajectories corresponding to the individual nuclei along the constant driving force line. (Such trajectories have been used to identify nuclei as "crystal-like" or "liquidlike" in previous work. ${ }^{3}$ ) It appears that as one moves toward the critical point, the nature of nuclei changes from what has been identified as crystal-like into what has been named as liquidlike. Indeed, a liquid skirt develops around the crystal, which can be characterized by a gradual transition between the composition of the crystal and that of the initial liquid (Fig. 6). The thickness of this liquid skirt seems to diverge toward $T_{c}$. As the transition between the crystal-like and liquidlike solutions is

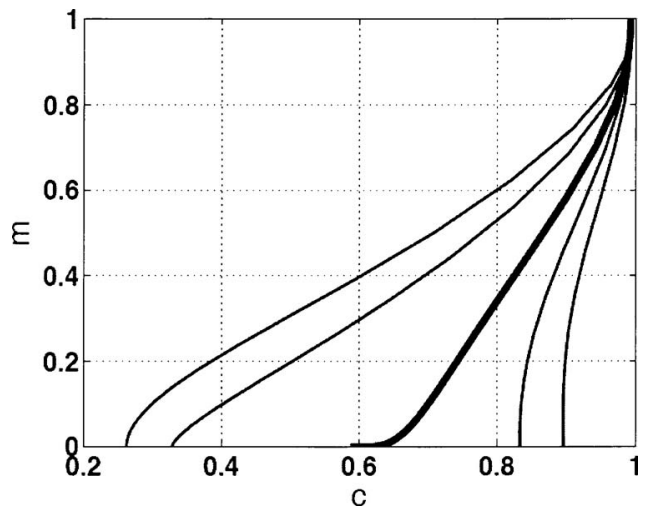

FIG. 6. Structural order parameter vs composition trajectories for points along the constant driving force line for the $\mathrm{Cu}$ rich solution. From left to right: $T / T_{c}=0.95,0.975,1.0,1.025$, and 1.05. Note the gradual transition from crystal-like nuclei into liquidlike, when approaching the metastable critical point $\left(T / T_{c}=1.0\right)$ from both sides.
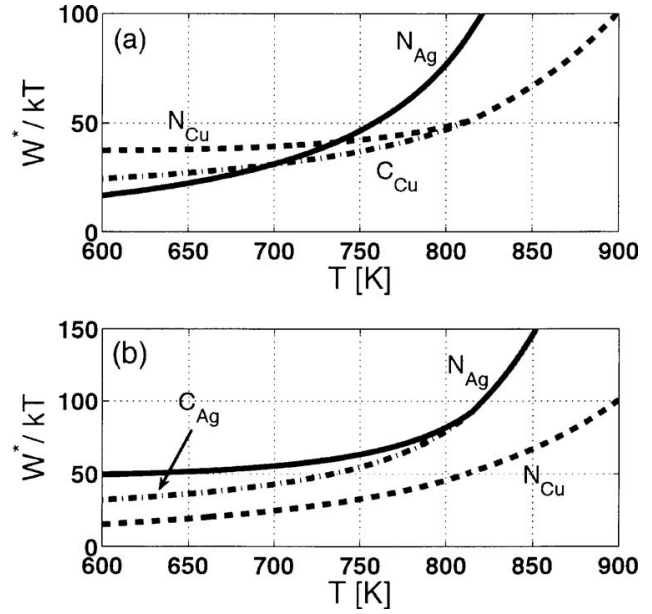

FIG. 7. Free energy for various nucleation pathways as a function of temperature (a) on the left and (b) on the right of the critical composition. [Notation: $N_{\mathrm{Ag}}$ and $N_{\mathrm{Cu}}$ stand for normal solutions, while $C_{\mathrm{Ag}}$ and $C_{\mathrm{Cu}}$ for composite solutions that are rich in the component sown in the subscript, respectively. Solid and dashed lines: $W^{*} / k T$ for the $\mathrm{Ag}$ and $\mathrm{Cu}$ rich normal solutions, respectively, observed when descending in the phase diagram at the critical composition, and following the coexistence line below $T_{c}$ (trajectories denoted by heavy black lines and arrows in Fig. 1). Dash-dot lines: $W^{*} / k T$ for the composite solutions along the bifurcation line (the trajectories denoted by heavy gray lines and arrows in Fig. 1)].

fairly gradual, one cannot easily identify any definite border line/transition zone between the two types. These findings are fully consistent with previous results from continuum models. We believe, however, that a full understanding of the maximum in the nucleation rate observed in colloidal systems along the coexistence line within the framework of such continuum models warrants further research and probably a full dynamical treatment of the problem,

\section{Crystal nucleation inside the spinodal region}

Inside the spinodal region the liquid phase is unstable with respect to concentration fluctuations and soon separates into two liquids of the compositions on the opposite sides of the metastable liquid-liquid coexistence line. Once large enough to accommodate the "normal" crystalline nuclei described in Part I, the phase transition inside these liquid regions of equilibrium composition takes place as described in Part I for the coexistence line. We denote these solutions of the EL equation as $N_{i}$, where subscript $i=\mathrm{Ag}$ or $\mathrm{Cu}$ indicates the majority component of the nucleating crystalline phase. Descending at the critical composition as shown in Fig. 1, we have both types of solutions above the critical temperature $\left(T>T_{c}\right), N_{\mathrm{Ag}}$ and $N_{\mathrm{Cu}}$. These solutions continue below $T_{c}$ along the coexistence lines on both the left and the right sides. The corresponding nucleation barrier heights are shown in Figs. 7(a) and 7(b), respectively. On the Ag rich side of the coexistence line, the $N_{\mathrm{Cu}}$ wins down to $\sim 740 \mathrm{~K}$. Below this temperature the solution $N_{\mathrm{Ag}}$ is preferred (has the lower free energy of formation). In contrast, on the $\mathrm{Cu}$ rich side of the coexistence line, the $\mathrm{Cu}$ rich solution $N_{\mathrm{Cu}}$ has the lowest free energy at all temperatures. Comparing all solutions on the two sides, the $\mathrm{Cu}$ rich solution $N_{\mathrm{Cu}}$ on the $\mathrm{Cu}$ rich side has the minimum nucleation barrier. Accordingly, well below $T_{c}$ and inside the spinodal region, crystal nucle- 


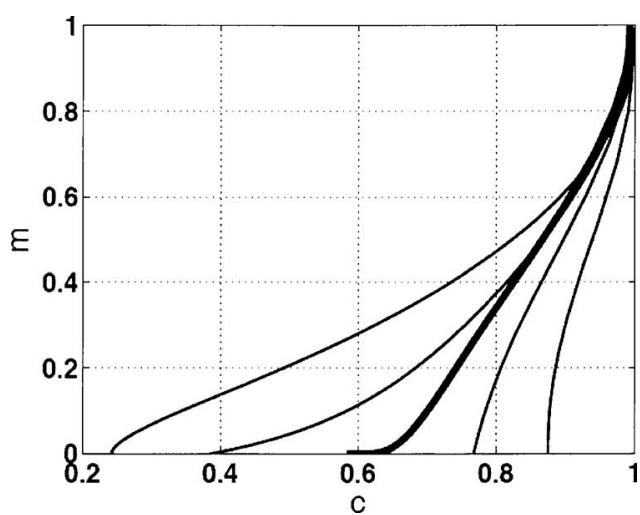

FIG. 8. Structural order parameter vs composition trajectories for points along the coexistence line for the $\mathrm{Cu}$ rich solution. From left to right: $T / T_{c}=0.85,0.95,1.0,0.95$, and 0.85 . Note the gradual transition from crystal-like nuclei into liquidlike, when approaching the metastable critical point $\left(T / T_{c}=1.0\right)$ from both sides.

ation is expected to happen in two stages, (i) first spinodal decomposition of the liquid takes place, which is followed by (ii) the formation of $\mathrm{Cu}$ rich normal nuclei $N_{\mathrm{Cu}}$ in the $\mathrm{Cu}$ rich liquid phase. Whether this stays so in the vicinity of the critical point, where a critical slowing down of the liquid phase separation is expected, remains an open question. To answer this question, one has to solve the full time dependent problem for spinodal decomposition and nucleation, and is, thus, out of the scope of the present paper, which addresses only the nucleation properties. An investigation along these lines would also raise fundamental questions, yet unanswered, including quantitative modeling of the kinetics of phase separation close to $T_{c}$ and deep inside the coexistence region. ${ }^{12}$ Finally, we wish to draw attention to the fact that both normal nuclei $N_{\mathrm{Ag}}$ and $N_{\mathrm{Cu}}$ become liquidlike when $T$ $\rightarrow T_{c}$ (Fig. 8).

\section{Crystal nucleation between the spinodal and coexistence lines}

First, we address crystal nucleation between the spinodal and coexistence lines at a temperature well below the critical point: $T=650 \mathrm{~K}$ (see Fig. 9). The composition range between the coexistence line and spinodal line can be divided into two regimes: One lying between the coexistence line, and what we call a "bifurcation" line that falls between the coexistence and spinodal lines (see region I and bifurcation lines in Fig. 1) and another lying between the bifuration and spinodal lines (region II in Fig. 1). Such a region appears on both sides of the liquid-liquid coexistence domain. Inside these regions, we can distinguish three different kinds of crystal nuclei for the same temperature and composition. For example on the Ag rich (left) side we observe the following.

(i) The continuation of normal $\mathrm{Ag}$ rich nuclei $\left(N_{\mathrm{Ag}}\right)$ into this region, discussed in Part I in detail.

(ii) The continuation of normal $\mathrm{Cu}$ rich nuclei $\left(N_{\mathrm{Cu}}\right)$.

(iii) "Composite" nuclei (henceforth, $C_{\mathrm{Cu}}$ ) that have a $\mathrm{Cu}$ rich crystalline center, and a liquid skirt whose $\mathrm{Cu}$ concentration falls between those of the central part and the initial liquid. In the vicinity of the liquidliquid coexistence line, the skirt becomes very well
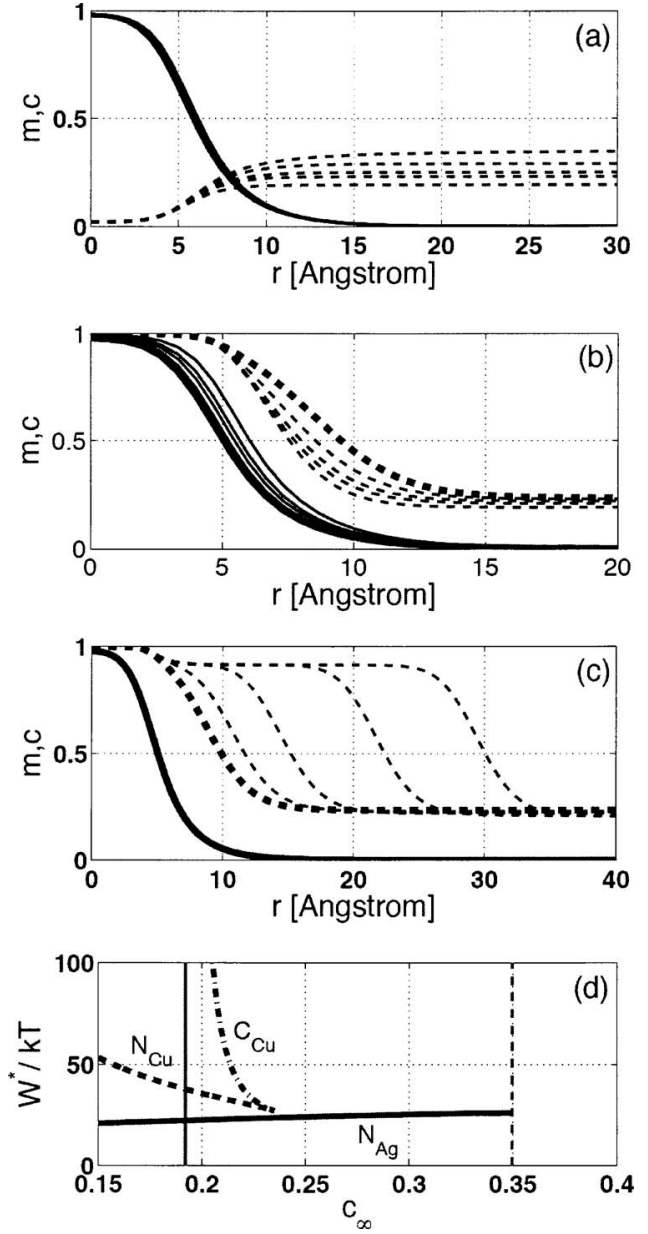

FIG. 9. Properties of $\mathrm{Cu}$ rich normal and composite nuclei forming at $T$ $=650 \mathrm{~K}$ : Shown are the radial phase field (solid) and the composition profiles (dashed) for (a) the Ag rich normal, (b) the $\mathrm{Cu}$ rich normal, and (c) the $\mathrm{Cu}$ rich composite nuclei. The solutions at the bifurcation point are denoted by heavy lines. Note the well-defined liquid layer around the crystalline core forming at small supersaturations and the convergence of the two types to each other at the bifurcation composition. (d) The respective nucleation barriers. ( $N_{\mathrm{Ag}}$ and $N_{\mathrm{Cu}}$ stand for normal solutions, while $C_{\mathrm{Cu}}$ for the composite solution that are rich in the component in the subscript.)

pronounced. In fact, here liquid phase separation starts with the nucleation of a new liquid phase. Remarkably, when approaching the liquid-liquid coexistence lines from inside, this composite nucleus can be well approximated by placing that crystal nucleus to the center of this liquid nucleus, which is preferred at the composition appearing at the center of the liquid nucleus. As we move toward the spinodal though, the liquid nucleus shrinks faster than the crystal nucleus at the center, and such separation becomes impossible. Interestingly, when we increase further the supersaturation, this composite nucleus $\left(C_{\mathrm{Cu}}\right)$ and the $\mathrm{Cu}$ rich normal nucleus $\left(N_{\mathrm{Cu}}\right)$ converge with each other at a bifurcation line. Beyond this supersaturation (regions II in Fig. 1), these solutions $\left(N_{\mathrm{Cu}}\right.$ and $\left.C_{\mathrm{Cu}}\right)$ disappear, and only the Ag rich solidlike nucleus $\left(N_{\mathrm{Ag}}\right)$ may exist. It is worth mentioning that crystal nuclei $N_{\mathrm{Ag}}$ and $N_{\mathrm{Cu}}$ also gain a long concentration "tail" when entering the liquid-liquid coexistence domain. The respective phase field and concentration 


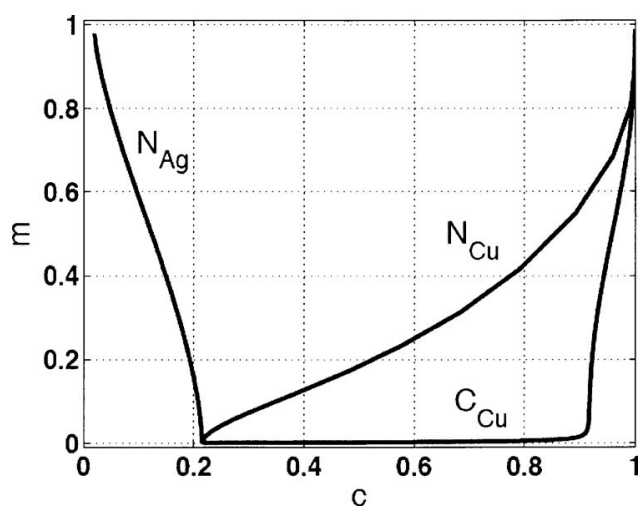

FIG. 10. Structural order parameter vs composition trajectories for the three types of solutions shown in Fig. 9, at the initial liquid composition of $c$ $=0.215$. Note the long horizontal line for the composite solution that represents the liquid layer around the solid core.

profiles and nucleation barrier heights are compared for these nucleation pathways in Fig. 9. An analogous behavior can be seen on the $\mathrm{Cu}$ rich side, however, there the normal nuclei $N_{\mathrm{Ag}}$ and composite nuclei $C_{\mathrm{Ag}}$ merge at the bifurcation line, while $N_{\mathrm{Cu}}$ exists in both regions I and II. This behavior resembles the nucleation pathways seen in a Cahn-Hilliard-type theory using a free energy with three parabolic minima, where "broad interface" and "thin interface" composite nuclei were seen to converge with each other at a critical undercooling. ${ }^{11}$ Apparently, on the Ag rich side of the coexistence region $N_{\mathrm{Cu}}$ and $C_{\mathrm{Cu}}$ play the role of thin and broad interface composite nuclei reported in Ref. 11, as opposed to the other side, where $N_{\mathrm{Ag}}$ and $C_{\mathrm{Ag}}$ are the analogs of these composite nuclei. The $\phi$ vs $c$ trajectories corresponding to the three nuclei possible at $650 \mathrm{~K}$ in region I on the left are compared in Fig. 10. A remarkable feature of the composite nucleus is an extended horizontal section in this plot representing a fully liquid layer of continuously changing composition.

The relation among these nucleation pathways depends on temperature. The nucleation barrier heights computed at $T=650,750$, and $800 \mathrm{~K}$ are shown in Figs. 11(a)-11(c), respectively, while the corresponding radial field profiles are presented in Figs. 12(a)-12(c). On the $\mathrm{Cu}$ rich side of the coexistence region the $N_{\mathrm{Cu}}$ is preferred at all temperatures. In contrast, on the $\mathrm{Ag}$ rich side, the nucleation barriers for $N_{\mathrm{Ag}}$ and $N_{\mathrm{Cu}}$ intersect each other $\left(W_{N_{\mathrm{Ag}}}=W_{N_{\mathrm{Cu}}}\right)$, either outside of the coexistence region or inside region I, so that $N_{\mathrm{Cu}}$ is preferred between this intersection composition $c_{N N}(T)$ and the left bifurcation line (see Fig. 13). Furthermore, in a temperature range between $T_{c}>T>\sim 690 \mathrm{~K}$, a similar crossing line exists between $N_{\mathrm{Ag}}$ and $C_{\mathrm{Cu}}\left(W_{N_{\mathrm{Ag}}}=W_{C_{\mathrm{Cu}}}\right)$, denoted by $c_{\mathrm{NC}}(T)$ in Fig. 13. Between $c_{\mathrm{NC}}(T)$ and the left bifurcation line, even the composite nucleus $C_{\mathrm{Cu}}$ is preferred to $N_{\mathrm{Ag}}$ though (with the exception of the bifurcation line) $N_{\mathrm{Cu}}$ is always preferred to $C_{\mathrm{Cu}}$.

It is also interesting to map the composition dependence of the minimum nucleation barrier at different temperatures below and above $T_{c}$ (Fig. 14). While for $T>T_{c}$ the behavior
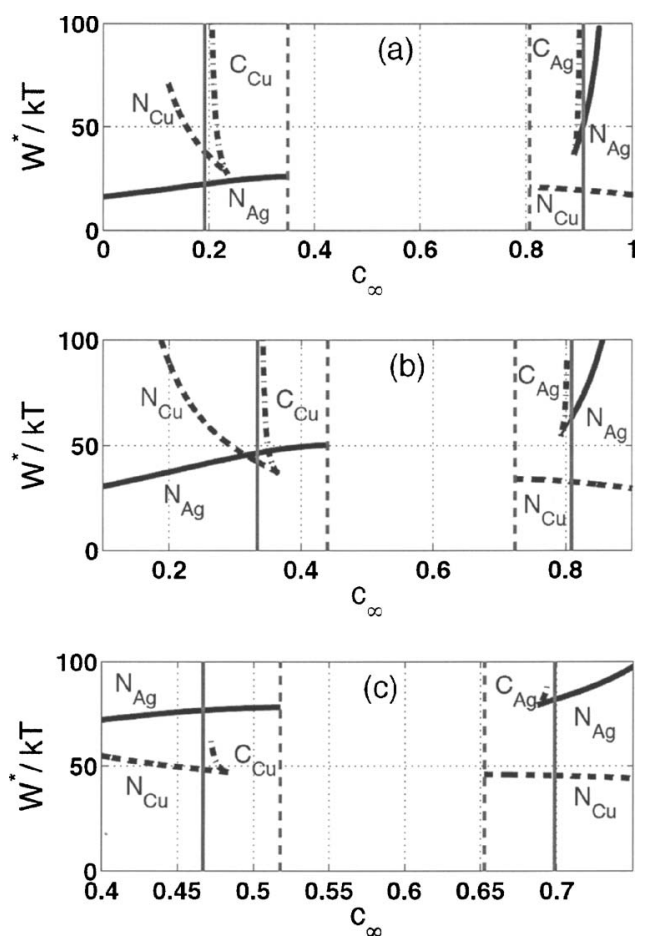

FIG. 11. Nucleation barrier vs initial liquid composition for the three types of solutions existing on the left and right of the critical composition at (a) $T=650 \mathrm{~K}$, (b) $T=750 \mathrm{~K}$, and (c) $T=800 \mathrm{~K}$. The coexistence and spinodal compositions are denoted by vertical solid and dashed lines, respectively.

is quite simple: either $N_{\mathrm{Ag}}$ or $N_{\mathrm{Cu}}$ dominates, a rather complex behavior is observed below $T_{c}$, where different nuclei may dominate in regions I and II and in the spinodal regime. The new type of composite nuclei are expected to be competitive only in a narrow composition range near the bifur-
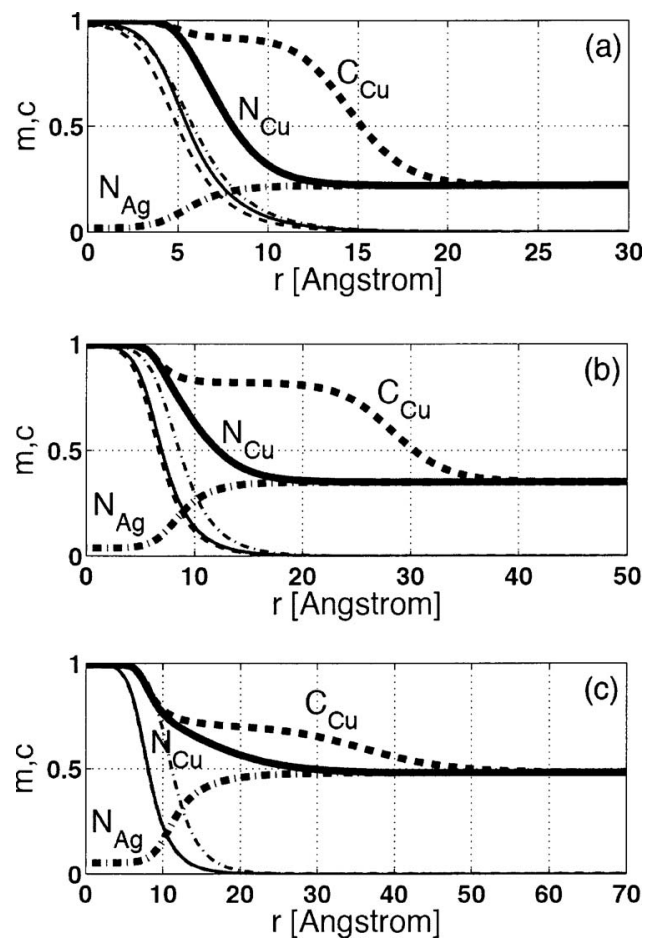

FIG. 12. Radial phase field (light lines) and composition (heavy lines) profiles for the three types of solutions existing on the left of the critical composition at (a) $T=650 \mathrm{~K}$, (b) $T=750 \mathrm{~K}$, and (c) $T=800 \mathrm{~K}$. 


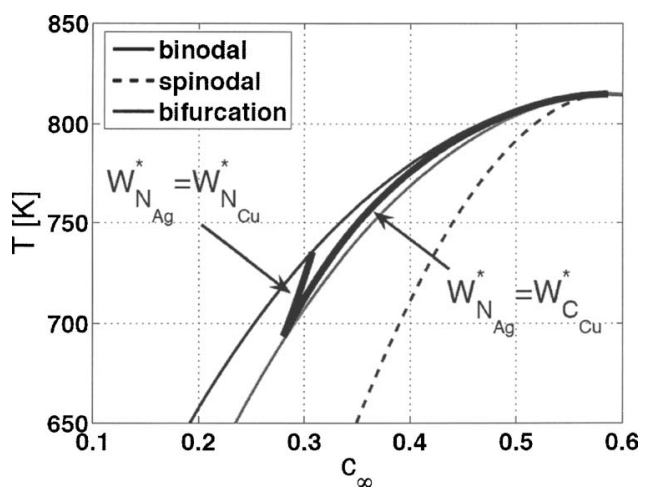

FIG. 13. Lines representing equal nucleation barrier heights for the $\mathrm{Ag}$ and $\mathrm{Cu}$ rich normal solutions $\left[c_{\mathrm{NN}}(T)\right.$ in the text $]$ and for the $\mathrm{Ag}$ rich normal solution and the $\mathrm{Cu}$ rich composite solutions $\left[c_{\mathrm{NC}}(T)\right.$ in the text]. Note that they terminate in a common point falling on the bifurcation line.

cation line, where the difference between the free energies of the two merging solutions is comparable to $k T$, but then the two solutions are very similar in every respect, so one cannot distinguish them in practice.

We are convinced that the same type of approach might help us to clarify phase preference in other systems with metastable critical point, and that the analogs of the composite nuclei and the bifurcation line exist in such systems, in general.

Finally, we wish to stress that these results address crystalline phase selection entirely on the basis of the nucleation barrier height, an approximation that is expected to be reasonable for metallic systems, where possible changes in the nucleation prefactor are expected to be negligible with respect to the changes in the nucleation barrier height. This is, however, not necessarily true in systems where there are compounds in whose vicinity the molecular mobility may change considerably, ${ }^{21}$ or in systems where the components have significantly different diffusivities/chemical diffusion is the rate limiting factor. ${ }^{22} \mathrm{~A}$ full dynamic phase field model, incorporating fluctuations into the equations of motion for all fields, is needed to address crystal nucleation in the latter cases. Work is underway into this direction.

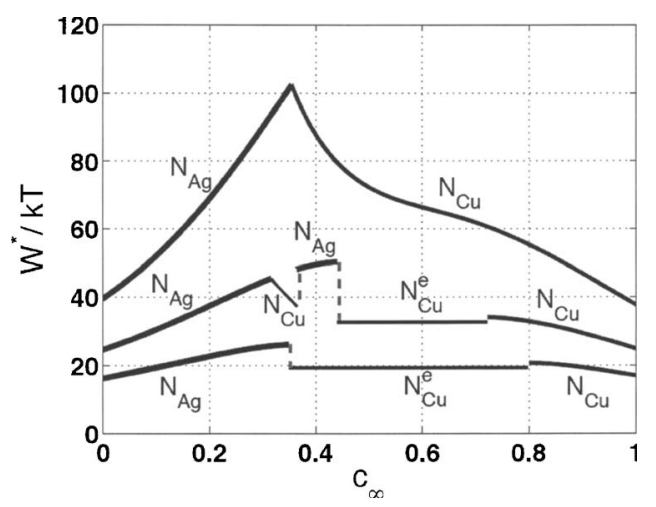

FIG. 14. Phase selection in the $\mathrm{Ag}-\mathrm{Cu}$ system, according to the minimum of the nucleation barrier at $T=850 \mathrm{~K}$ (upmost curve), $750 \mathrm{~K}$ (central curve), and $650 \mathrm{~K}$ (bottom curve). Note the complex behavior below the critical point. $\left(N_{\mathrm{Ag}}\right.$ and $N_{\mathrm{Cu}}$ stand for normal solutions that are rich in the component denoted by the subscript, while $N_{\mathrm{Cu}}^{e}$ denotes the normal solution forming on the $\mathrm{Cu}$ rich branch of the coexistence line.)

\section{SUMMARY}

As part of our study of homogeneous crystal nucleation in eutectic systems, we have investigated possible nucleation pathways in the metastable liquid-liquid miscibility gap occurring below the eutectic temperature and in the vicinity of the respective metastable liquid-liquid critical point. We have shown the following.

(i) Three pairs of nuclei may occur inside the liquidliquid coexistence region. Liquid-liquid nuclei $(\mathrm{Ag}$ rich and $\mathrm{Cu}$ rich); normal crystal-liquid nuclei (again $\mathrm{Ag}$ rich and $\mathrm{Cu}$ rich) that are the continuation of the nuclei seen above the critical point; and composite nuclei ( $\mathrm{Ag}$ rich and $\mathrm{Cu}$ rich), in which the solid core is surrounded by a well defined liquid layer resembling to the liquid-liquid nucleus forming at the initial liquid composition.

(ii) Of these nuclei, in principle, four types may occur on both the left and right of the spinodal line. These are (a) a liquid-liquid nucleus rich in the minority component of the initial liquid and three kinds of possible crystal nuclei. Two solutions that have a core that is rich in the minority component: (b) one that is the continuation of the normal nuclei seen above $T_{c}$ and (c) another composite nucleus in which the crystalline core appears inside a well defined liquid droplet resembling the liquid-liquid nucleus forming at that initial liquid composition. The last one (d) is the normal crystal nucleus whose crystal core is rich in the majority component of the initial liquid. Remarkably, moving toward the spinodal line from outside, solutions (b) and (c) converge to each other at a bifurcation line. Between this line and the spinodal line only (a) and (d) may exist.

(iii) An enhanced nucleation rate is expected near the critical point for both the $\mathrm{Ag}$ and $\mathrm{Cu}$ rich crystal nuclei along lines for which the driving force is constant. This is in agreement with previous theoretical work, molecular simulations, and some of the experiments performed on crystallizing liquids with metastable fluid-fluid coexistence.

\section{ACKNOWLEDGMENTS}

This work has been supported by the Hungarian Academy of Sciences under contract No. OTKA-K-62588 and by the ESA PECS Nos. 98021 and 98043.

${ }^{1}$ G. I. Tóth and L. Gránásy, J. Chem. Phys. 127, 074709 (1997).

${ }^{2}$ P. R. Len Wolde and D. Frenkel, Science 277, 1975 (1997); Theor. Chem. Acc. 101, 205 (1999); Phys. Chem. Chem. Phys. 1, 2191 (1999); A. Lomakin, N. Asherie, and G. B. Benedek, Proc. Natl. Acad. Sci. U.S.A. 100, 10254 (2003).

${ }^{3}$ (a) V. Talanquer and D. W. Oxtoby, J. Chem. Phys. 109, 223 (1998); (b) R. P. Sear, ibid. 114, 3170 (2001); (c) D. W. Oxtoby, Philos. Trans. R. Soc. London, Ser. A 361, 419 (2003); (d) A. Shiryayev and J. D. Gunton, Philos. Trans. R. Soc. London, Ser. A 120, 8318 (2004).

${ }^{4}$ R. M. L. Evans, W. C. K. Poon, and M. E. Gates, Europhys. Lett. 38, 595 (1997); K. G. Soga, J. M. Melrose, and R. C. Ball, J. Chem. Phys. 110, 2280 (1999).

${ }^{5}$ O. Galkin and P. G. Vekilov, Proc. Natl. Acad. Sci. U.S.A. 97, 6277 (2000); W. Pan, A. B. Kolomeisky, and P. G. Vekilov, J. Chem. Phys. 
122, 174905 (2005).

${ }^{6}$ R. P. Sear, J. Phys.: Condens. Matter 19, 033101 (2007).

${ }^{7}$ W. Hu and D. Frenkel, Macromolecules 37, 4336 (2004).

${ }^{8}$ For example, A. Kose and S. Hachisu, J. Colloid Interface Sci. 55, 487 (1976); C. Smits, J. S. van Duijneveldt, J. K. Dhoni, H. N. W. Lekkerkerker, and W. J. Briels, Phase Transitions 21, 157 (1990); D. Rosenbaum, P. C. Zamora, and C. F. Zukoski, Proc. Am. Acad. Arts Sci. 76, 150 (1996); D. Rosenbaum and C. F. Zukoski, J. Cryst. Growth 169, 752 (1996)

${ }^{9}$ C. R. Berland, G. M. Thurston, M. Kondo, M. L. Broide, J. Pande, O. O. Ogun, and G. B. Benedek, Proc. Natl. Acad. Sci. U.S.A. 89, 1214 (1992); N. Asherie, A. Lomakin, and G. B. Benedek, Phys. Rev. Lett. 77, 4832 (1996); M. L. Broide, T. M. Tomic, and M. D. Saxowsky, Phys. Rev. E 53, 6325 (1996); M. Muschol and F. Rosenberger, J. Chem. Phys. 107, 1953 (1997).

${ }^{10}$ For example, C. D. Cao, G. P. Görler, D. M. Herlach, and B. Wei, Mater. Sci. Eng., A 325, 503 (2002).

${ }^{11}$ L. Gránásy and D. W. Oxtoby, J. Chem. Phys. 112, 2410 (2000).

${ }^{12}$ J. D. Gunton, M. San Miguel, and P. S. Sahni, in Phase Transitions and Critical Phenomena, edited by C. Domb and J. L. Lebowitz (Academic Press, London, 1983) Vol. 8, p. 267.

${ }^{13}$ N. Inaba, K. Sato, S. Suzuki, and T. Hashimoto, Macromolecules 19,
1690 (1986); P. Schaaf, B. Lotz, and J. C. Wittmann, Polymer 28, 193 (1987).

${ }^{14}$ A. H. Ramsden and P. F. James, J. Mater. Sci. 19, 1406 (1984); J. Mater. Sci. 19, 2894 (1984); E. D. Zanotto, P. F. James, and A. F. Craievich, ibid. 21, 3050 (1986).

${ }^{15}$ A. Cacciuto, S. Auer, and D. Frenkel, Phys. Rev. Lett. 93, 166105 (2004).

${ }^{16}$ L. Gránásy, T. Pusztai, D. Saylor, and J. A. Warren, Phys. Rev. Lett. 98, 035703 (2007).

${ }^{17}$ J. W. Cahn and J. E. Hilliard, J. Chem. Phys. 28, 258 (1958).

${ }^{18}$ W. H. Press, S. A. Teukolsky, W. T. Wetterling, and B. P. Flannery, Numerical Recipes in C (Cambridge University Press, Cambridge, 1992).

${ }^{19}$ Z. Chvoj, S. Srikanth, and P. Ramachandrarao, J. Non-Equilib. Thermodyn. 24, 360 (1999). The thermodynamic assessment has been done by P. R. Subramanian and J. H. Perepezko, [J. Phase Equilib. 14, 62 (1993)].

${ }^{20}$ J. W. Cahn and J. E. Hilliard, J. Chem. Phys. 31, 688 (1959).

${ }^{21}$ L. Battezzati and A. L. Greer, Acta Metall. 37, 1791 (1989).

${ }^{22}$ A. L. Greer, P. V. Evans, R. G. Hamerton, and D. K. Shangguan, J. Cryst. Growth 99, 38 (1990); Z. Kozisek and P. Demo, ibid. 132, 491 (1993); K. F. Kelton, Philos. Mag. A 361, 429 (2003); Acta Mater. 48, 1967 (2000). 